\title{
VARIATIONS ANATOMIQUES \\ CHEZ DISTOMUM CLOACICOLA MAX LÜHE 1909
}

\author{
Par Robert-Ph. DoLLFUS
}

Parmi les nombreuses espèces de Distomes parasites de la couleuvre à collier, Natrix natrix (L. 1754), qui ont été décrites, trois d'entre elles n'ont pas encore reçu une attribution générique conforme à la systématique actuellement en usage, ce sont : Distomum assula F. Dujardin 1845, Dist. gracillimum Max Lühe 1909, et Dist. cloacicola Max Lühe 1909. Dans la présente note il sera question seulement de cette troisième espèce, que Lühe n'a pas figurée et qu'il trouva en Allemagne.

Les premières description et figure d'un distome adulte chez Natrix et correspondant à la deseription de cloacicola par Lühe (1909, p. 121-122) sont celles publiées par Agostino Rizzo (1902, p. 29-30, fig. 2) d'après les individus trouvés dans la dernière partie de l'intestin à Catane. Rizzo désigna ces individus sous le nom de "Distomum acervocalciferum Gastaldi », les considérant comme la forme adulte d'une métacercaire qu'il trouva enkystée sous la peau des Rana esculenta L. de Catane et Paterno (Sicile), mais dont il ne donna pas la description.

Rappelons que «Distoma acervocalciferum 》 Biagio Gastaldi (1854, p. 28-29, 35-36, pl. I, fig. VI-IX) est une métacercaire progénétique (œufs dans l'utérus) observée très fréquemment par Gastaldi à Turin, enkystée le long des trones nerveux du plexus brachial des Rana esculenta L. Aucune dimension n'a été indiquée par Gastaldi, et les figures qu'il en a données sont trop peu précises pour qu'il soit possible de savoir si e'est bien la même espèce qui a été trouvée en Sicile par Rizzo.

Chez les spécimens de Rizzo $(\hat{1}, \vec{b} \times 0,5 \mathrm{~mm}$.), la ventouse orale (diamètre 0,30 ) est un peu plus grande que la ventrale (diam. 0,25 ); il y a un très court œesophage et, conformément à la description de Lühe, les cæca intestinaux n'atteignent pas l'extrémité postérieure du corps, se terminant avec le quatrième cinquième de la longueụr du corps. Les testicules sont décrits comme symétriques et figurés

Ann. de Parasitologie, T. XXV, $\mathrm{x}^{\circ} 3 .-1950$, p. 141-149. 
presque symétriques ; l'ovaire est entre le testicule droit et le bord postérieur de l'acetabulum; les vitellogènes sont au niveau de l'acetabulum, en partie ventralement aux cæca, en partie un peu

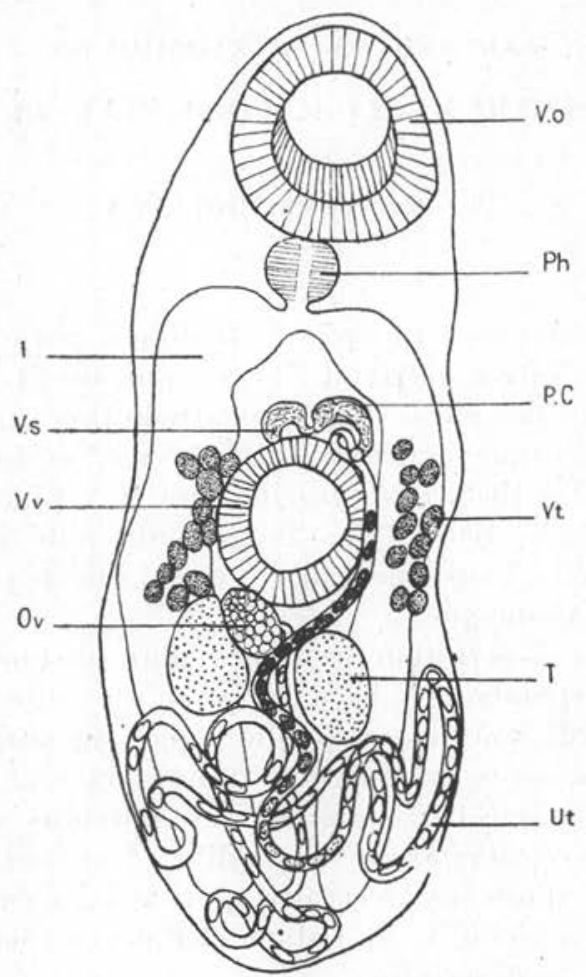

Fıg. 1. - Paralepoderma cloacicola (Max Lühe 1909) de Natrix natrix (L.), Italie ; dessin exécuté sous la direction de J.-G. Baer. I, intestin ; Ov, ovaire ; $\mathrm{PC}$, poche du cirre; $\mathrm{Ph}$, pharynx ; T, testicule ; Ut, utérus ; Vs, vésicule séminale externe; $\mathrm{Vt}$, vitellogènes; Vv, ventouse ventrale; Vo, ventouse orale. La vessie excrétrice n'est pas figurée.

en dedans des cæca. Les œufs sont dits mesurer $9-13 \times 6 \mu$, ce qui est certainement une erreur.

Gustav Mödlinger (1924, p. 196, pl. fig. 1) a décrit et figuré comme "Distomum cloacicola Lühe » des spécimens à testicules exactement symétriques, qu'il trouva dans le cloaque de $N$. natrix (L.) en Hongrie. Ces spécimens sont conformes à la description de Lühe, 
sauf que les cæca intestinaux atteignent l'extrémité postérieure du corps et que l'ovaire est contre le côté droit de l'acetabulum, ce qui ne s'accorde pas avec la description de Rizzo, mais l'on peut admettre une certaine variabilité individuelle pour la longueur des cæca et pour l'emplacement de l'ovaire.

Une forme qui paraìt bien ètre morphologiquement identique, ou presque, et que, dans mes notes manuscrites, j'ai autrefois étiquetée « Lepoderma cloacicola (Max Lühe) 》, est la métacercaire progénétique trouvée chez Planorbis planorbis (L.) par le regretté Pierre Paris à Saint-Jean-de-Losne (Cóte-d'Or), que j'ai étudiée en 1932 (voir en particulier 1932, p. 409-411, fig. 2-4), mais je ne l'ai pas considérée comme un vrai Lepoderma (=Plagiorchis $)$ s. str. pour trois raisons. D'abord parce que je n'ai pas vu de spinules cuticulaires (seulement une striation cuticulaire), ensuite parce que les testicules sont symétriques ou subsymétriques, enfin parce que la vessie en Y ne passe pas entre les testicules (1). La première de ces trois raisons n'est pas valable, parce que les spinules pouvaient être tombées lorsque le matériel a été mis en eau formolée, et l'on sait que, chez quelques espèces de Lepoderma, les spinules sont très caduques et ne sont observables que sur le vivant. En ce qui concerne la deuxième et la troisiéme raisons, je les tiens pour valables, car, chez les Lepoderma typiques, les testicules ne sont jamais symétriques et la partie impaire de la vessie passe toujours entre les deux testicules.

Chez mes métacercaires progénétiques de Planorbis, les cæca intestinaux se terminent plus ou moins loin dans le troisième quart de la longueur du corps, sans pénétrer dans le dernier quart (différence avec la description de Mödlinger), l'emplacement de l'ovaire est un peu variable, son centre étant à un niveau compris entre ceux du bord postérieur et du centre de l'acetabulum. Pour les œufs, les dimensions ne sont pas constantes : $33,55 \times 18,34 \times 18,36 \times 19$, $38,5 \times 19 \mu$; à maturité ils contiennent un miracidium bien discernable, paraissant achevé ou presque achevé.

Des distomes que je crois aussi référables à Dist. cloacicola Lühe ne sont pas rares dans le cloaque ou l'intestin postérieur des Natrix natrix (L.) en Italie et en France. Plusieurs spécimens ont été récoltés dans l'intestin postérieur de natrix de la région de Bologne (Italie) par J.-G. Baer qui m'a aimablement offert une figure inédite

(1) J'ai dit (1932, p. 411) que le trone impair de la vessie passe entre les testicules et se bifurque immédiatement après ; c'est une erreur, chez la métacercaire à maturité il ne passe pas entre les testicules et se bifurçue immédiatement avant ou à leur niveau, 
(fig. 1) d'un de ces spécimens. On remarquera que les testicules ne sont pas absolument symétriques, que l'ovaire a son centre un peu en arrière du niveau du bord postérieur de l'acetabulum, que les cæca intestinaux s'avancent postérieurement plus loin que ne l'ont

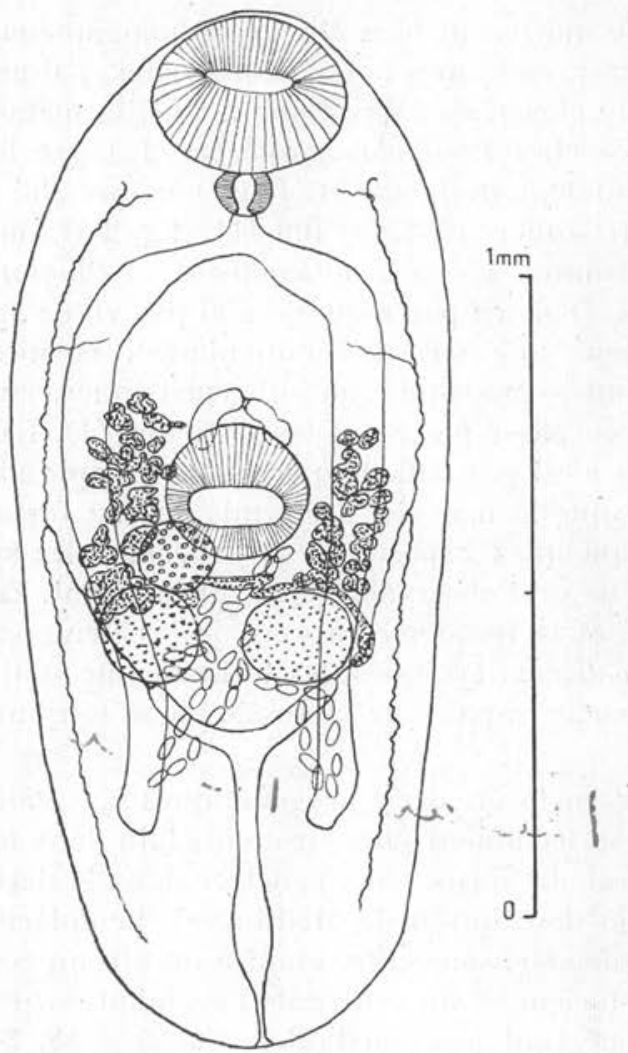

FIG. 2. - Mème espèce, de Natrix natrix persa (Pallas), région de Bologne (Italie) ; Ipse legi et delin., 4-6-1938.

figuré Rizzo (1902, fig. 2) et moi-même (1932, fig. 4), presque aussi loin que l'a figuré Mödlinger (1924, fig. 1).

Lors de la dissection, au laboratoire de Richelieu, de quelques N. natrix persa (Pallas, 1811) d'Italie, qui m'avaient été procurées par mon ami Emile Brumpt, j'ai aussi trouvé (23-8-1934 et 4-6-1938), soit dans le cloaque, soit entre le cloaque et la fin de l'intestin moyen, des distomes atteignant au maximum un peu plus de $2 \mathrm{~mm}$. ; 
je les ai considérés comme des cloacicola. L'examen sur le vivant m'a permis de constater la présence de spinules cuticulaires sur le

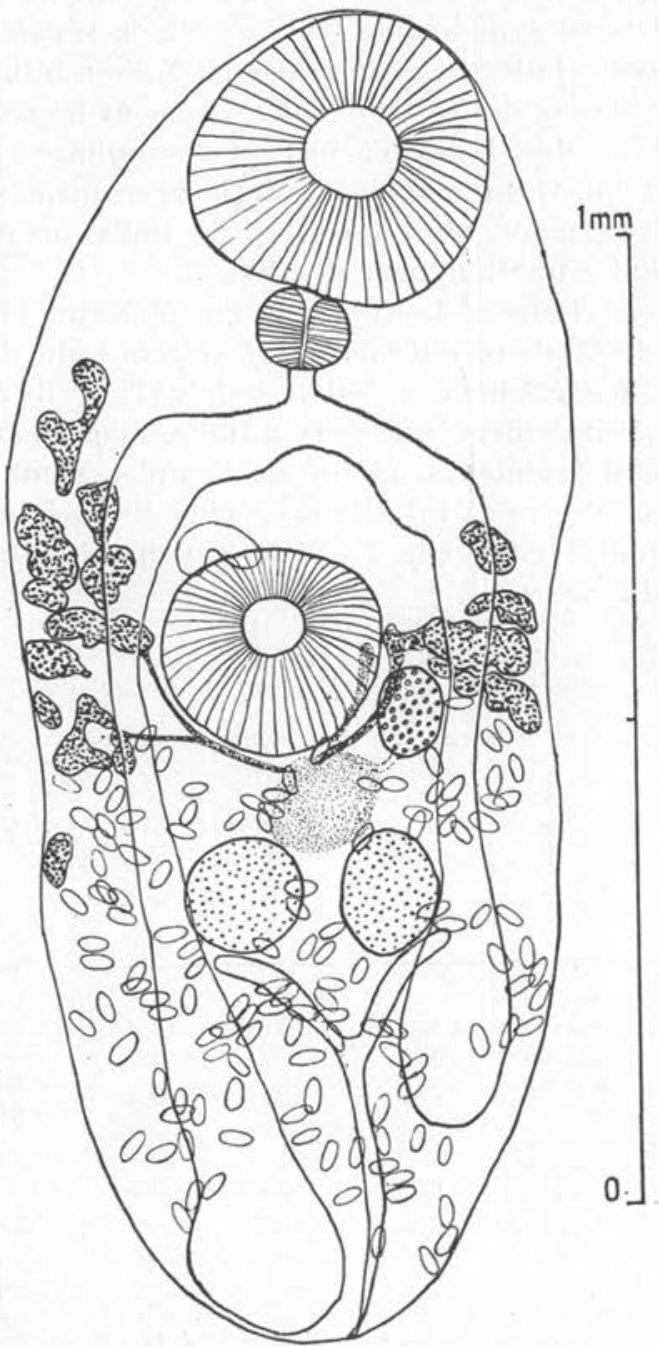

Fig. 3. - Même espèce, de Natrix natrix (L.) ; Richelieu (Indre-et-Loire) ; Ipse legi et delin., 13-8-1932. Individu vu par la face dorsale.

quart antérieur du corps, mais ces spinules sont tombées intégralement aussitôt que les distomes ont été traités par un fixateur ou 
mis en alcool. J'ai noté que les cæca intestinaux se terminaient à mi-distance du bord posiérieur de l'acetabulum et de l'extrémité postérieure du corps; chez tous mes spécimens, les testicules étaient exactement symétriques (còte à côte ou séparés par un petit espace où passait l'utérus) et la vessie en Y avec bifurcation immédiatement en arrière des testicules (fig. 2) ; pour les œufs, j'ai trouvé 29-30 $\times 16-17 \mu$. Max Lühe n'a pas vu d'œesophage ; j'ai remarqué que, chez les individus à région antérieure contractée, l'œsophage n'était pas discernable, alors que chez les individus en extension il pouvait atteindre une longueur de $105 \mu$.

A Richelieu (Indre-et-Loire), j'ai eu plusieurs fois l'occasion (depuis août 1932) de récolter des D. cloacicola Lühe dans le cloaque ou l'intestin postérieur des Natrix natrix (L.) ; ils étaient un peu plus petits, généralement, que ceux d'Italie, mais montraient clairement une aussi grande variabilité de l'emplacement de l'ovaire et de la longueur des cæca intestinaux; chez un spécimen, le cæcum droit était plutôt court (fig. 3) et le gauche atteignait l'extrémité postérieure du corps.

Dimensions en mm. (sauf pour les oufs)

\begin{tabular}{|c|c|c|c|c|c|c|}
\hline & 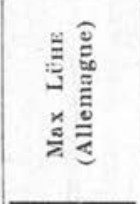 & 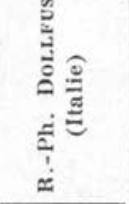 & 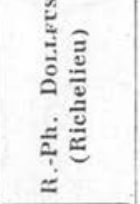 & 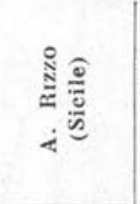 & \multicolumn{2}{|c|}{$\begin{array}{c}\text { Ch. Joyeux et J. Gaud } \\
\text { (Maroc) }\end{array}$} \\
\hline Longueur....... & $1,3-1,4$ & $1,83-2,0$ & 1.41 & $1,5-2,0$ & $1,10-1,40$ & $1,90-2,60$ \\
\hline Largeur......... & $0,5-0,6$ & 0,51 & 0,54 & 0,5 & $0,5-0,6$ & $0,5 \cdot 0,7$ \\
\hline Ventouse orale. & $\begin{array}{r}0,30-0,32 \\
\times 0,32-0,35\end{array}$ & 0,31 & 0,31 & 0,30 & $\begin{array}{r}0,23-0,29 \\
\times 0,09-0,26\end{array}$ & $0,28 \times 0,312$ \\
\hline $\begin{array}{r}\text { Ventouse ven - } \\
\text { trale........... }\end{array}$ & $\begin{array}{r}0,20-0,27 \\
\times 0,25-0,35\end{array}$ & 0,23 & 0,23 & 0,25 & $0,175-0,200$ & $0,240.0,300$ \\
\hline $\begin{array}{l}\text { Pharynx ........ } \\
\text { Esophage...... }\end{array}$ & $\begin{array}{c}0,11 \\
0\end{array}$ & $\begin{array}{c}0,088 \\
0,05-0,10\end{array}$ & $\begin{array}{l}0,105 \\
0,036\end{array}$ & $\stackrel{?}{\text { présent }}$ & $\begin{array}{c}0,08-0,105 \\
\text { présent } \\
\text { jusqu'à } \\
0,065\end{array}$ & $\begin{array}{l}0,12-0,13 \\
\text { présent }\end{array}$ \\
\hline GEufs........... & $\begin{array}{r}28-30 \\
\times 15,17 \mu\end{array}$ & $\begin{array}{r}29-30 \\
\times 16 \cdot 17 \mu\end{array}$ & $\begin{array}{c}31 \times 17 \\
\text { a } 34 \times 20 \mu\end{array}$ & $\begin{array}{l}(9-13 \\
\times 6 \\
\times 6) ? ?\end{array}$ & $28 \times 17 \mu$ & $\begin{array}{c}30-31 \\
\times 18-20 \%\end{array}$ \\
\hline
\end{tabular}

La même espèce a été redécrite comme Lepoderma cloacicola (Lühe) d'après des individus de l'intestin de Natrix (= Tropidonotus) viperinus (Latreille, 1802), de $\mathrm{Si}$ Allal Tazi (Maroc), par 
Ch. Joyeux et J. Gaud (1945, p. 112-115), malheureusement sans figure. Ces auteurs ont insisté sur la variabilité individuelle de certains caractères : œsophage absent lorsque l'individu n'est pas en extension, longueur des cæca intestinaux, niveau atteint antérieurement et postérieurement par les vitellogènes, emplacement de l'ovaire par rapport aux testicules et à l'acetabulum. Les variations qu'ils ont constatées sont les mêmes que celles présentées par nos spécimens d'Italie et de Richelieu.

Au cours de l'été dernier (juillet-octobre 1949), Alice Buttner a trouvé, à Richelieu (Indre-et-Loire), chez Planorbis planorbis (L.), une xiphidiocercaire leptocerque dont elle avait vu la métacercaire dans des têtards d'Alytes obstetricans (Laurenti) et Triton palmatus (Schneid.) de la même localité. Cette cercaire montrait un court prépharynx qui a disparu chez la métacercaire mûre ; celle-ci devenait toujours progénétique. D’après sa morphologie, cette métacercaire correspond, à très peu près, à Paralepoderma cloacicola (Lühe) ; elle en diffère par son ovaire plus gros et généralement un peu plus antérieur, une dégénérescence précoce des testicules, des œufs un peu plus gros $(40 \times 20 \mu)$. L'œsophage est bien visible, les cæca intestinaux n'atteignent pas l'extrémité postérieure du corps et se terminent vers le niveau de la bifurcation de la vessie.

Expérimentalement, Alice Buttner a obtenu la pénétration et l'enkystement chez des têtards d'Alytes obstetricans (Laurenti), des têtards d'Amblystoma mexicanum (Shaw) et de jeunes Xenopus laevis (Daudin). Toutes ces métacercaires devenaient progénétiques. Des œufs de celles-ci ont éclos dans le tube digestif de nombreux Planorbis planorbis (L.) qui les avaient ingérés. Dans l'intestin postérieur des Natrix natrix (L.) de Richelieu, A. Buttner a trouvé (septembre 1949) des Paralepoderma cloacicola (Lühe) adultes et a estimé incertaine l'identité spécifique de ceux-ci avec les métacercaires progénétiques trouvées par elle dans la nature et obtenues expérimentalement. Mlle Buttner a été amenée à conclure plutôt à la non-identité spécifique parce qu'ayant fait ingérer, à deux reprises, par des natrix, de nombreuses métacercaires enkystées de tous àges, elle constata, au bout d'environ 6 jours, la dégénérescence ou, la disparition de toutes les métacercaires non progénétiques et la simple survivance de quelques-unes des progénétiques.

J'ai personnellement examiné, à Richelieu, les cercaires, métacercaires et adultes étudiés par Alice Buttner; je crois probable, contrairement à l'opinion d'Alice Buttner, que les cercaires et métacercaires appartiennent, comme les adultes, à cloacicola, mais je n'en ai pas acquis la certitude ; il se pourrait que les différences tiennent à des conditions biologiques particulières. 
Discussion. - Dist. cloacicola Lühe ressemble quelque peu à certaines espèces de Glypthelmins à testicules symétriques ou subsymétriques, mais les vrais Glypthelmins n'ont pas la vessie en Y et sont des Brachycaliidæ alors que Dist. cloacicola Lühe appartient aux Lepodermatidæ (= Plagiorchiidæ). Parmi les nombreux genres de cette dernière famille, il n'y en a aucun, à ma connaissance, chez lequel se trouvent réunis les deux caractères :

1) Testicules symétriques ou subsymétriques.

2) Vessie en Y ne passant pas entre les testicules, se bifurquant en arrière des testicules.

J'estime done qu'en raison de caractères aussi importants, il est nécessaire de proposer un nouveau genre : Paralepoderma n. gen., qui est défini ainsi :

"Caractères généraux des Lepoderma, sauf en ce qui concerne la position des testicules, qui sont symétriques ou subsymétriques, et la position de la vessie, qui ne passe pas entre les testicules et se bifurque immédiatement en arrière d'eux.

«Espèce-type : Paralepoderma cloacicola (Max Lühe, 1909). »

La description de Dist. cloacicola Lühe, par Lühe, devra être complétée et un peu modifiée pour tenir compte des caractères suivants : cuticule spinulée antérieurement, à spinulation extrêmement caduque, observable seulement sur le vivant; œsophage visible chez les individus en extension, où il peut atteindre environ la longueur du pharynx ; cisca intestinaux se terminant à un niveau variant entre la fin du troisième quart de la longueur du corps et l'extrémité postérieure du corps ; vessie en Y se bifurquant immédiatement en arrière des testicules ; ovaire entre le testicule droit et l'acetabulum, ou dorsal à l'acetabulum contre son bord postérieur droit ; vitellogènes dépassant antérieurement parfois l'acetabulum ; receptaculum seminis absent ; canal de Laurer presque sûrement présent; poche du cirre petite contre le bord antérieur de l'acetabulum ; longueur des œufs 28 à $34 \mu$, largeur 16 à $20 \mu$.

Cycle évolutif supposé. - Premier hôte : Planorbis. Deuxième hôte : têtard de Batracien (1). Hc̀tes définitifs : Natrix natrix (L.), N. n. persa (Pallas), N. viperinus (Latreille). Allemagne, France, Italie, Maroc.

(1) Supposition fondée sur le fait que les Natrix sont prédatrices des têtards et sur mon interprétation des expériences poursuivies pendant l'été de 1949 par Alice Buttner. (Voir A. Buttner, 1950, p. 235-236 et 1950, p. 21-26). 


\section{Bibliographie}

Buttien (Alice), - Prımière démonstration expérimentale d'un cycle abrégé chez les Trématodes digénétiques (Plagiorchis brumpti n. sp.). C.R. Acad. Sc., CCXXX, 9-1-1950, 235-236.

Première démonstration expérimentale d'un eycle abrégé chez les Trématodes digénétiques. Cas du Plagiorehis brumpti n. sp. Annales Parasitol., XXV, 1950, p. 21-26.

Dolifus (Robert-Ph.). - Métacercaire progénétique chez un Planorbe. Ann. Parasitol., Paris, X, 1932, 407-413, fig. 1-4.

Gastald (Biagio). - Cenni sopra alcuni nuovi elminti della Rana esculenta con nuove osservazioni sul Codonocephalus mutabilis (Diesing). Tesi per aggregazione Collegio Fac. Sc. R. Univ. Torino, $4^{\circ}$, 1854, p. 25-36, pl. I, fig. I-IX, pl. II, fig. I-IV.

Joveux (Charles) et GAUn (Jean). - Recherches helninthologiciues marocaines. Archives Institut Pasteur. Maroc, Casablanea, III, 1945, 111-143, fig. 1-6.

Lüне (Max). - Trématodes. Süssıvasserfauna Deutschlande, Heft. 17, Jena 1909 , p. 1-217, fig. 1-188.

Mönlinger (Gustav). - Neue Trematoden der Ungarischen Fauna. Mat. és Term. -tud Ertesitö. XLI lï̈tet. III. tábla. 1924; p. 193-197 + 1 p., pl. fig. 1-5.

Írzzo (Agostino). - La fauna elmintologica dei Rettili nella provincia di Catana. Archives Paresitol., Paris, VI, 1 ; 25-7-1902, p. 26-41, fig. 1-11. 